\title{
Intolerance to Prone Positioning as a Clinical Marker of Motor \\ Delay in Infants
}

\begin{abstract}
Background and objectives: The literature over the past decade has shown associations between lack of prone positioning (tummy time) and motor delays. In our clinical practice, we observed that parents of infants with motor delays often reported their children were excessively fussy during tummy time. Our study was designed to explore the association between excessive fussiness in prone and the presence of motor delays.
\end{abstract}

Methods: Data from 303 patients under age 3 presenting to the Early Intervention program at an academic medical cente over a two year period were reviewed retrospectively. Historical information on length and tolerance of prone positioning in infancy and developmental diagnosis (Motor Delay, Language Delay, Global Developmental Delay, Autism Spectrum Disorder (ASD) and No Delay were included).

Results: Both measures of intolerance to prone position (time in prone and fussiness in prone) showed significant linear associations with developmental diagnosis. Specifically, likelihood of never fussing (X2 (1) $=19.1, p<0.001$ ) and of experiencing $>$ five minutes of prone position daily (X2 (1)=13.1, $p<0.001)$ were significantly lower in subjects who had motor delays than in subjects with other delays and no delays. There was no significant difference in fussing and time in prone among children with other developmental diagnoses, including language delay, global developmental delay, autism spectrum disorder (ASD).

Conclusion: There is an association between the degree of fussiness in infants when placed in prone and the presence of motor delays. Parent report of excessive fussiness may be a useful clinical tool for identifying increased risk of motor delay in infants.

\section{Abbreviations}

ASD: Autism Spectrum Disorder; BTS: Back To Sleep; SIDS: Sudden Infant Death Syndrome; DP: Deformational Plagiocephaly; EIP: Early Intervention Program; SES: Socioeconomic Status; PDMS Peabody Developmental Motor Scale; AIMS: Alberta Infant Motor Scale; DAYC: Developmental Assessment of Young Children; CARS: Clinical Autism Rating Scale; ADOS: Autism Diagnostic Observation Scale; PT: Physical Therapy

\section{Introduction}

The prevalence of gross motor delays in infants appears to have increased over the past two decades and several studies have suggested that a lack of prone positioning of infants when awake is associated with an increased rate of gross motor delays [1-4]. These studies used parent report of night time and daytime positioning and then performed standardized motor assessments during the first year of life, which revealed significantly lower scores in those infants that slept supine and spent less time in prone when awake. One study of healthy full term newborns also looked at milestone acquisition and found that prone milestones were delayed in supine sleepers but walking was not [2]. These studies did not specifically look what barriers there were to the prone position or the infants' ability to

\section{Journal of}

Pediatrics \& Child Care

\author{
Jennifer Cross ${ }^{1 *}$, Dawnnica Eastman², Sarah Brovender ${ }^{3}$ \\ and Mary $\mathrm{J}$ Ward $^{1}$
}

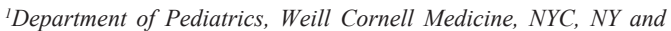
Division of Child Development, Komansky Center for Children's Health at NY Presbyterian Hospital, New York, USA

${ }^{2}$ Cape Cod Hospital, Hyannis, MA 02601, USA

${ }^{3}$ Stony Brook School of Medicine, Stony Brook, NY, USA

*Address for Correspondence:

Jennifer Cross, Department of Pediatrics, Komansky Center for

Children's Health, Helmsley Medical Tower 505 East 70th Street 3rd

Floor, New York, NY 10021, USA, E-mail: jfc2001@med.cornell.edu

Submission: 05 May, 2017

Accepted: 06 June, 2017

Published: 13 June, 2017

Copyright: (c) 2017 Cross J, et al. This is an open access article distributed under the Creative Commons Attribution License, which permits unrestricted use, distribution, and reproduction in any medium, provided the original work is properly cited.

tolerate that position. This study was designed to investigate the role of intolerance to prone positioning in infancy and how that might contribute to motor delays.

Recent research has indicated a marked increase in the number of children presenting with deformational plagiocephaly (DP), and congenital torticollis over the past decade; supine sleep position and lack of prone time were the most significant associated factors for these disorders [5-8]. Children who present with DP have been shown in a longitudinal study of over 200 infants to demonstrate a higher prevalence of motor delay during infancy [9]. Although a smaller study by Kennedy et al. did not find the association between higher rates of motor delay in a group of infants with DP compared to controls, the study was consistent with prior findings in showing an association between lack of time in prone and lower scores on motor assessments in both the DP and control group [10].

In 1992, The American Academy of Pediatrics (AAP) began the 'Back to Sleep' (BTS) campaign to reduce the incidence of sudden infant death syndrome (SIDS). The AAP recommended that all infants be placed supine for sleep, which led to a decrease in the overall amount of time infants spent in prone [11]. The BTS campaign was highly successful in decreasing the rate of SIDS [12,13], but did not initially provide recommendations to attend to prone positioning during wakeful time. This omission was felt to be a contributing factor to the observed increase in motor delays [14].

In 2006, with an increasing awareness of the importance of early detection of developmental delays, the AAP Council on Children with Disabilities made recommendations for developmental surveillance at each well child visit and standardized screening of all children at 9 , 18 and 30 months to screen for possible developmental delays [15]. In 2008 , the AAP revised the BTS guidelines to include recommendations for frequency and duration of supervised prone positioning of infants when awake. These included starting 'tummy time' immediately after birth for 3 to 5 minutes, 2 to 3 times a day [16]. However despite these 
recommendations, it appeared that infants still may not be exposed to adequate time in the prone position, and that there continued to be inadequate awareness of the AAP recommendations among practitioners and parents [17,18]. In 2013 the AAP published a new algorithm for the surveillance and early screening of motor delays in infants [19]. In the introduction to this publication, the authors describe responses from a focus group of pediatricians at the AAP National Conference in 2010. The group surveyed was unsure of how to most effectively assess and manage infants with motor delays and requested standardized recommendations. This appeared to be one of the impetuses for the development of the new algorithm.

In assessing motor skills, it is well established that motor development follows a predictable sequence in a cephalo-caudal distribution which mirrors the head to toe maturation of the central nervous system. Head control is one of the earliest motor skills to develop and is fundamental for the development of trunk control which is a prerequisite for sitting, crawling and walking. From a clinical perspective it is important to identify motor delays early, not only to begin therapy but also to identify children with global delays including autism spectrum disorder (ASD). The first indication of a global developmental problem is often the presence of a motor delay [20]. The new motor algorithm from the AAP provides a framework for early identification of these motor delays, as well as identification of neuromuscular abnormalities, such as cerebral palsy or hemiplegia [19].

In a developmental pediatrics practice at an academic medical center, providers observed that children with significant motor delays were reported to be extremely intolerant of the prone position in infancy. Although there are reports in the literature that indicate that fussiness is a barrier to prone positioning $[3,17]$, there appear to be no studies that have examined specifically the issue of fussiness as a marker for motor delay. This study was designed to investigate the association between parent report of infant response to prone positioning and its association with motor delay. We hypothesized that children with motor delays would be reported to have been more intolerant of prone positioning in infancy than children with other types of delay and children with typical development. Excessive fussiness could then be a useful clinical tool for early detection of motor delays in the office or clinic setting. This could help raise awareness among health care providers, that an infant with excessive fussiness may have an underlying motor delay or be at higher risk for plagiocephaly. This study adds a rationale for using history or observation of excessive fussiness in prone as a clinical marker for motor delay at all early infant visits.

\section{Methods}

A retrospective review was conducted using consecutive records from an early intervention program (EIP) at an academic medical center for January 2010 through December 2012. Children in this program are eligible for evaluation and services regardless of family income and immigration status. Therefore ethnicity and socioeconomic status (SES) were not recorded in the clinic charts, as mandated by state law. As a proxy for SES, demographic information was collected using Zip Code ${ }^{\mathrm{pw}}$ and US Census data to describe ranges of income and ethnicity [21]. The sample included subjects from the metropolitan area for which the center had a contract. Information on median income was obtained from the US Census Bureau data for 2010. In this sample, $74 \%$ of subjects lived in areas with incomes above the median for the area; only $6 \%$ of subjects lived in areas with median income below the city poverty threshold [20]. In total, $47 \%$ percent of subjects lived in Zip Code ${ }^{\mathrm{m}}$ areas with white populations above $70 \%$ and $13 \%$ lived in Zip Code ${ }^{\mathrm{m} m}$ areas that were over $70 \%$ Black and Hispanic. All children were between the ages of 1 and 36 months.

Prior to evaluation, each parent completed a history questionnaire about the child to aid in the assessment. Developmental testing using standardized instruments was conducted and combined with clinical observations and developmental history to complete the evaluation.

The review yielded 400 records. Some subjects had multidisciplinary assessments elsewhere and were referred solely to this hospital site for audiology assessment, feeding evaluation, or other subspecialty evaluation and so did not complete the intake questionnaire. Other records were missing data on tolerance to prone positioning due to parent omission. The distribution of diagnoses for those subjects who did not have questionnaire information was similar to those who did.

Thus, all records with a completed questionnaire including data on parent report of prone positioning were included in analyses, resulting in a sample of 303 subjects. No subjects were excluded for any reason other than incomplete information. As part of the intake questionnaire, parents were asked two questions: 1$)$ Time: total time in prone per day before age 6 months (0-5 minutes, 5 to 15 minutes, $>15$ minutes). 2) Tolerance: response to prone position before age 6 months (always, sometimes, never fussed).

The following data were extracted from the record: birth history including gestation age and birth weight, gender, milestone history, standard scores from developmental tests, medical diagnosis, therapy services recommended, and parent report regarding tolerance of and time spent in prone before 6 months of age. Developmental diagnoses were obtained from a multidisciplinary assessment, which employed standardized assessment tools, providing standard scores. Clinicians used this information and clinical impression to assign a medical diagnosis with appropriate ICD 9 codes that were accepted by the EIP. The most common medical diagnoses were hypotonia/ hypertonia with motor delay, language delay, global developmental delay and autism spectrum disorder, those that did not qualify for the program were diagnosed as no delay. As this was a hospital setting, many subjects were referred from the hospital neonatal intensive care unit (NICU) follow-up clinic. For children born prematurely, standard scores were based on corrected age (i.e. chronological age minus the number of months premature). Corrected ages were used until the age of 24 months, at which point chronological age was used.

The standardized instruments used to measure motor development were the Peabody Developmental Motor Scale (PDMS) or the Alberta Infant Motor Scale (AIMS). Diagnosis of global developmental delay was made based on delays in two or more domains, one of which included the cognitive domain, using standardized developmental tests such as the Development Assessment of Young Children (DAYC). Language delays, which could be an isolated language delay or part of a more global delay, were assessed using the Preschool Language Scale - Fourth Edition (PLS-4). Diagnosis of autism was 
Citation: Cross J, Eastman D, Brovender S, Ward MJ. Intolerance to Prone Positioning as a Clinical Marker of Motor Delay in Infants. J Pediatr Child Care. 2017;3(1): 06

ISSN: 2380-0534

made using a standardized instrument, either the Clinical Autism Rating Scale (CARS) or the Autism Diagnostic Observation Schedule (ADOS).

Both clinical diagnoses and standard scores were used to classify developmental delays for purposes of this report. A classification of motor delay was defined as a primary medical diagnosis of motor delay with hypotonia or hypertonia for which physical therapy (PT) was recommended. Where dual diagnoses (e.g. motor delay and global developmental delay) were given, subjects were classified as being in the motor delay group if the gross motor standard score was $>2.0$ standard deviations (SD) below the mean. When the child had autism and global developmental delay without significant motor delay the diagnosis was categorized as autism. Developmental diagnoses for this study thus included: motor delay, isolated language delay, global developmental delay, autism or no delay.

Data were analyzed using IBM SPSS statistics (version 22). Categorical data were analyzed using $X^{2}$ and Spearman rankorder correlations. Continuous data were analyzed using Pearson's correlation and ANOVA with post-hoc comparisons utilizing the Bonferroni correction $(\mathrm{p}<0.05)$.

\section{Results}

\section{Developmental diagnosis}

Figure 1 presents the distribution of diagnoses in this sample based on criteria outlined above. Motor delays constituted a plurality of diagnoses $(n=110 ; 36 \%)$. A large portion $(n=81 ; 27 \%)$ of these referred subjects did not qualify for a diagnosis based on Early Intervention criteria. The EIP criteria used at our institution were the same as the local state and federal regulations, which established the following definitions for eligibility: a delay more than 2 standard deviations below the mean in one domain on a standardized developmental test, or a delay of between 1.5 and 2 standard deviations below the mean in two or more developmental domains. Certain medical diagnoses such as extreme prematurity, autism or genetic syndromes associated with developmental delay qualify for services based on the diagnosis as well as any associated delays [22]. As the clinic is a referral center, rates of ASD were high $(n=55 ; 18 \%)$. Isolated language delays were observed in $10 \%$ of cases $(n=30)$ and global developmental delay with language and cognitive delay, without significant motor impairment or autism, was observed in $9 \%(\mathrm{n}=27)$.

\section{Subjects}

Average age in the sample $(n=303)$ subjects was 19.6 months $(S D=8.3)$, with a range of 3 to 38 months. Average birth weight ( $n=300$ ) was 2.95 kilograms ( $S D=0.8$ ), with a range of 0.52 to $4.62 \mathrm{~kg}$. Table 1 provides information about the subjects included in the study sample, based on diagnosis groups specified by study hypotheses. Analyses indicated that age at evaluation $(F[2,300]=13.4, p<0.001)$, birth weight $(F[2,299]=18.5, p<0.001)$, and child gender $\left(X^{2}[2]=12.2\right.$, $p<0.01)$ differed significantly by diagnosis group. Specifically, for age at assessment, subjects in the Other Delays group were significantly older than subjects in the No Diagnosis and Motor Delay groups. For birth weight, subjects in the Motor Delay group had significantly lower birth weights than those in the other two groups, due to the higher proportion of premature infants in the motor delay group.
Among children in the autism group there were a higher proportion of males as would be expected, but in motor delay group, and in the non-delayed group there was an approximately equal gender split.

\section{Parent report of tolerance to prone position}

In this sample, $26 \%$ of parents reported no fussiness in response to prone positioning, 51\% reported at least some fussiness, and $23 \%$ reported that the infant always fussed in prone position. Parent reports of time spent in prone position suggest reasonable compliance with AAP recommendations: $49 \%$ of parents reported at least 15 minutes per day of prone position in infancy; an additional 39\% reported 5 to 15 minutes per day and only $12 \%$ reported less than five minutes per day in prone. Parent report of time in prone was significantly correlated with reported fussiness in prone $(r[291]=0.42, p<0.001)$ indicating that infants who were reported to be more fussy also were reported to have spent less time in prone. Using Cohen's standard a Spearman correlation of 0.42 would qualify as a medium association [23]. If the convention of squaring the correlation coefficient is used, the proportion of variance shared between the two measures of prone position response is $18.4 \%$. Thus, parental recall of time spent in prone each day shares variance with recall of degree of fussiness in prone, but the two measures overlap at only a medium level.

\section{Developmental diagnosis and prone behavior}

Both measures of intolerance to prone position (time in prone and fussiness in prone) showed significant linear associations with developmental diagnosis using non-parametric analyses. Specifically, likelihood of never fussing $\left(X^{2}(1)=19.1, p<0.001\right)$ and of experiencing five or more minutes of prone position daily $\left(X^{2}(1)=13.1, p<0.001\right)$ were significantly lower in subjects who had motor delays than those with other delays, without significant motor delays, and those with

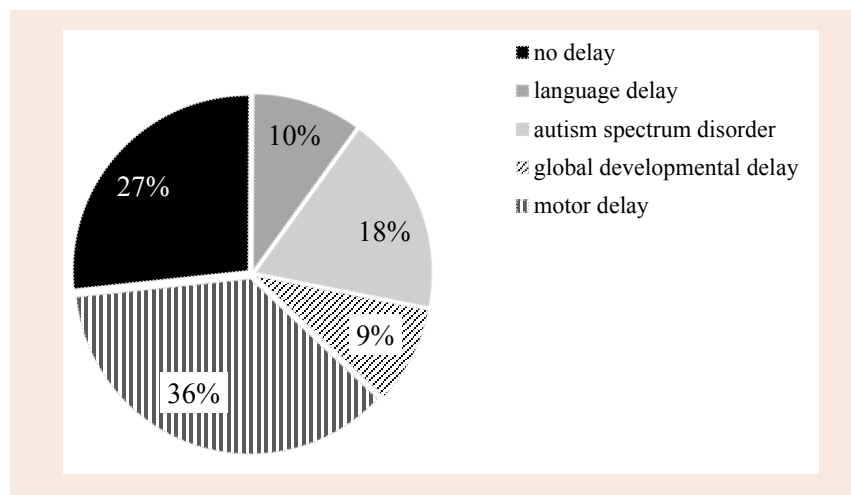

Figure 1: Distribution of developmental diagnoses in the study sample $n=303$.

Table 1: Infants Evaluated through the Early Intervention Program (EIP) from 2010 to 2012.

\begin{tabular}{|c|c|c|c|}
\hline & $\begin{array}{c}\text { No Delay } \\
(n=81)\end{array}$ & $\begin{array}{c}\text { Other Delays } \\
(n=112)\end{array}$ & $\begin{array}{l}\text { Motor Delay } \\
\quad(n=110)\end{array}$ \\
\hline $\begin{array}{l}\text { Age at evaluation (months) } \\
\qquad \begin{array}{l}M(S D) \\
(n=303)\end{array}\end{array}$ & $\begin{array}{l}18.60 \\
(6.5)\end{array}$ & $\begin{array}{c}22.58 \\
(8.2)\end{array}$ & $\begin{array}{l}17.23 \\
(8.6)\end{array}$ \\
\hline $\begin{array}{l}\text { Birth weight (kilograms) } \\
M(S D) \quad(n=300)\end{array}$ & $\begin{array}{l}3.12 \\
(0.6)\end{array}$ & $\begin{array}{l}3.18 \\
(0.8)\end{array}$ & $\begin{array}{l}2.59 \\
(0.9)\end{array}$ \\
\hline $\begin{array}{c}\text { Gender (male) } \\
\mathrm{n}(\%) \quad(\mathrm{n}=303)\end{array}$ & $\begin{array}{c}50 \\
(0.62)\end{array}$ & $\begin{array}{c}85 \\
(0.76)\end{array}$ & $\begin{array}{c}59 \\
(0.54)\end{array}$ \\
\hline
\end{tabular}

$\mathrm{SD}=$ Standard Deviation from the average age or birth weight reported; Cell sizes vary based on missing data. 
no delay (Figures 2 and 3). It is also of note that rates of extreme fussiness were highest in the group of infants with motor delays. The associations were present when controlling for age of child at assessment and gestational age at birth using birth weight as a proxy for gestation age. Infants with birth weights below $2.5 \mathrm{~kg}(n=78)$ were not reported to be significantly more fussy than birth weights over 2.5 $\operatorname{kg}(n=225), p=0.4$.

We also evaluated eligibility status for PT. By EIP standards this requires gross motor skills, in the absence of other delays, to be $>2 S D$ below the mean, which included all the children in our motor group. As eligibility for PT also includes children with gross motor scores between $1.5 S D$ and $2 S D$ below the mean, if other delays are present, this group included some of the children who were in our autism and global developmental delay group. Total number receiving PT was larger than our primary motor group, $n=135$. We found that the larger group continued to show the same associations, specifically the likelihood of qualifying for and receiving physical therapy in our sample was significantly higher in those children that were reported to always or sometimes fuss in prone than for infants who never fussed $\left(X^{2}(1)=13.06, p<0.001\right)$.

\section{Discussion}

The findings in this study support the hypothesis that infants who are less tolerant of tummy time are more likely to receive a diagnosis of motor delay both as infants and when they are toddlers, than those whose parents reported minimal fussiness during prone positioning.

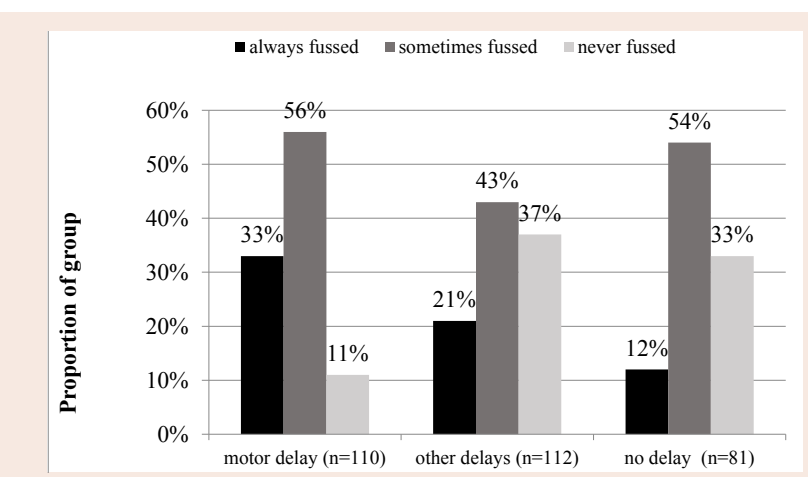

Figure 2: Report of fussiness in prone by diagnostic groups $(n=303)$. Note: Cell sizes vary based on missing data.

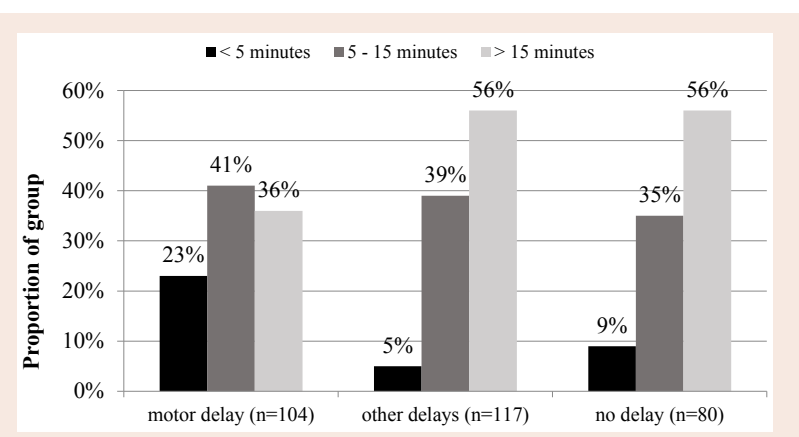

Figure 3: Report of time in prone per day by diagnostic groups $(n=301)$ Note: Cell sizes vary based on missing data.
Based on these findings, it is also true that infants reported to have spent less time in prone positioning were also more likely to have motor delays and that fussiness is a likely barrier to time in prone.

Although conclusions about direction of effects cannot be made using these data, it is clear that the cluster of increased fussiness and reduced time in prone were associated specifically with motor delays, rather than other developmental diagnoses, without a significant motor delay. As fussiness during tummy time may be a barrier to placing a child in prone, one explanation of the findings is that the motor delays are caused by the lack of prone positioning. It is possible that caregivers of infants who fuss during tummy time may be less likely to place them in prone, and more likely to hold them or position them where they are less fussy, such as a play mat or bouncy seat, leading to a lack of opportunity to develop motor skills such as head control and trunk control, as well as potentially exacerbating plagiocephaly due to lack of head movements.

Another explanation is that this group of infants could have some intrinsic neuromuscular deficits that make them particularly uncomfortable when in prone. This may be particularly true of our group in which subjects were already identified as having possible developmental delays and therefore represent a high risk sample. For example, in prone an infant has to work against gravity to extend the neck and turn his head to visually explore the environment. An inability to do this could cause distress and discomfort. This may then lead the infant to become fussy, and the natural response of the caregiver would be to pick the child up for comfort, thus ending the period of tummy time prematurely. We postulate that this lack of tummy time may then exacerbate the neuro-motor weaknesses in an already vulnerable infant and lead to increasing motor delays.

Lobo and Galloway showed that teaching parents specific handling techniques, including prone positioning, significantly improved scores on the AIMS of typically developing infants at 5 months compared to typically developing controls [24]. In the control group, parents were not given the positioning training and told, instead, to spend the time talking with their child in an infant seat. All the infants developed within normal ranges, but the intervention group acquired skills slightly earlier; for example, crawling in quadruped developed at 8.7 months in the control group and 7.5 months in the treatment group and walking, at 12.3 and 11 months, respectively. Given that appropriate handling and exposure to different positions in typical infants enhances motor development, it is likely that a lack of movement experiences, including lack of prone positioning may delay motor development, particularly in a more vulnerable population who already may have some motor abnormalities or developmental delays. We suggest that infants need to experience a variety of different physical positions to expose their developing nervous system to a range of sensory proprioceptive input and expose all muscle groups to the varying effects of gravity in different positions. We suspect that if infants do not have this exposure it may lead to muscle imbalance and weakness, which could then exacerbate distress in certain positions particularly in an infant who may already have a neuromuscular weakness.

An interesting prospective study by Carmeli et al. investigated the association between sleep positions and preferred awake and play positions in healthy full term infants at 3 and 6 months and looked at 
the relationship with AIMS scores [25]. A preferred position would meet criteria for being well tolerated by the infant and in this study, which did not find an association between motor delay and sleep position, they reported $60 \%$ of the infants 'preferred' the prone awake and play position at 3 months and $99 \%$ preferred it at 6 months. This would support the hypothesis that an infant who prefers prone and is tolerant of the position is less likely to have motor delays.

There is compelling research in early brain development suggesting that lack of experience leads to lack of development of skills due to poor synaptic connections between the sensory and motor cortex [26-28]. When the newborn brain is exposed to different stimuli it will begin to strengthen neuronal connections in the brain as well as pruning connections to neurons that are not stimulated [29]. Therefore, it is important to expose the infant to a variety of physical positions and proprioceptive input in order to strengthen the neuronal connections within the cortico-spinal system allowing for the appropriate advancement of postural motor development and by extension motor skills [30].

It is well established that postural control develops as a result of movement and experiences in a variety of positions [31]. When muscles work against gravity muscular strength increases [32]. When in prone the infant has to lift its head against gravity, thus improving head and neck control which are the precursors to the postural control necessary for all subsequent motor skills.

Thus it is reasonable to correlate lack of experience in prone with decreased exposure to the necessary positioning required to develop appropriate head movements against gravity, and to establish appropriate neck and trunk control need for typical motor development.

Results of this study show that children who spent less time in prone and were more fussy in prone, had a significantly higher rate of motor delays than infants who were not fussy and spent longer in prone. These findings suggest that fussiness in prone could be a useful marker for the early identification of infants with motor delays, particularly those who may be high risk for developmental delays, and this may have a practical application during routine developmental surveillance in the pediatricians office.

The AAP provides general recommendations regarding the amount and frequency of tummy time starting at the first newborn visit [16], however there are no specific guidelines on how a primary care provider might assess barriers to tummy time such as excessive fussiness in prone. We suggest that the primary care provider, such as the pediatrician or nurse practitioner could, during the well-baby assessment, place the infant in prone to see how they respond and ask parents if this is a typical or non-typical response. This could be done as early as the first newborn visit and thereafter, which gives the primary care practitioner the opportunity for anticipatory guidance regarding the importance of prone positioning. If the infant is persistently fussy in tummy time on subsequent visits, despite guidance regarding placing in prone, we would speculate that this infant may be at higher risk for a motor delay and may warrant referral to the EIP, a physical therapist or a developmental pediatrician for further evaluation.

There are study limitations that require further discussion. First, measures to quantify 'fussiness' in infants were based solely on parent report about their infants' behavior, and sometimes they were asked to recall the information as much as a year previously which would be subject to recall bias. In our analyses however, it was found that the rate of reported extreme fussiness was about the same for children who were both above and below 12 months of age at the time of evaluation. This finding suggests parents were able to recall details about their infants' behavior in early infancy. A second limitation is that our study included a selected group of children who had already been identified with possible delays. Although a significant portion was not determined to be delayed, a control group of typically developing infants was not available to assess fussiness.

This appears to be the first report to show that excessive fussiness in tummy time, in addition to lack of time in prone, may be a significant risk factor for motor delays in infants. We were able to show that infants who were reported to 'always' fuss in prone spent significantly less time in prone and had significantly higher rates of motor delays than infants who were reported to 'never' fuss. We had approximately a quarter of our study group that were reported to 'always' fuss, and a quarter who 'never' fussed, which allowed us to compare groups of similar sizes. With our study group we were also able to compare children with primarily motor delays to those with other delays, including autism as well to a group with no delays. One strength of this study is an adequate sample size of 303 subjects to test this hypothesis.

An area of future research is a prospective evaluation of prone behavior in infants followed in a primary care practice. This could confirm study findings that reports of excessive fussiness in a normative group is associated with suspected or actual motor delays during the first year of life. If poor tolerance to prone positioning proves to be a useful marker, primary care practitioners could begin to incorporate these simple questions and observations into their routine developmental surveillance during infancy and increase their identification of potential motor delays.

\section{References}

1. Majnemer A, Barr RG (2005) Influence of supine sleep positioning on early motor milestone acquisition. Dev Med Child Neurol 47: 370-376.

2. Kuo YL, Liao HF, Chen PC, Hsieh WS, Hwang AW (2008) The influence of wakeful prone positioning on motor development during the early life. J Dev BehavPediatr 29: 367-376.

3. Monson RM, Deitz J, Kartin D (2003) The relationship between awake positioning and motor performance among infants who slept supine. Pediatr Phys Ther 15: 196-203.

4. Salls JS, Silverman LN, Gatty CM (2002) The relationship of infant sleep and play positioning to motor milestone achievement. Am J Occup Ther 56: 577-580.

5. Argenta LC, David LR, Wilson JA, Bell WO (1996) An increase in infant cranial deformity with supine sleep positioning. J Craniofac Surg 7: 5-11.

6. Kane AA, Mitchell LE, Craven KP, Marsh JL (1996) Observations on a recent increase in plagiocephaly without synostosis. Pediatrics 97(6 Pt 1): 877-885.

7. Turk AE, McCarthy JG, Thorne CH, Wisoff JH (1996) The 'back to sleep campaign' and deformational plagiocephaly: is there a cause for concern? $\mathrm{J}$ Craniofacial Surg 7: 12-18.

8. Joganic JL, Lynch JM, Littlefield TR, Verrelli BC (2009) Risk factors associated with deformational plagiocephaly. Pediatrics 124: e1126- e1133.

9. Speltz ML, Collett BR, Stott-Miller M, Starr JR, Heike C, et al. (2010) Case-control study of neurodevelopment in deformational plagiocephaly. Pediatrics 125: e537-e542. 
Citation: Cross J, Eastman D, Brovender S, Ward MJ. Intolerance to Prone Positioning as a Clinical Marker of Motor Delay in Infants. J Pediatr Child Care. 2017;3(1): 06.

\section{ISSN: $2380-0534$}

10. Kennedy E, Majnemer A, Farmer JP, Barr RG, Platt RW (2009) Motor development of infants with positional plagiocephaly. Phys Occup Ther Pediatr 29: 222- 235.

11. (1992) American Academy of Pediatrics AAP Task Force on Infant Positioning and SIDS: Positioning and SIDS. Pediatrics 89(6 Pt 1): 1120 1126.

12. (1996) Positioning and sudden infant death syndrome (SIDS): update. American Academy of Pediatrics Task Force on Infant Positioning and SIDS. Pediatrics 98(6 Pt 1): 1216-1218.

13. (2000) Changing concepts of sudden infant death syndrome: implications for infant sleeping environment and sleep position. American Academy of Pediatrics. Task Force on Infant Sleep Position and Sudden Infant Death Syndrome. Pediatrics 105: 650-656.

14. Davis BE, Moon RY, Sachs HC, Ottolini MC (1998) Effects of sleep position on infant motor development. Pediatrics 102: 1135-1140.

15. Council on Children With Disabilities; Section on Developmental Behavioral Pediatrics; Bright Futures Steering Committee; Medical Home Initiatives for Children With Special Needs Project Advisory Committee (2006) Identifying infants and young children with developmental disorders in the medical home: an algorithm for developmental surveillance and screening. Pediatrics 118: $405-420$.

16. (2008) Back to sleep, tummy to play. American Academy of Pediatrics.

17. Zachry AH, Kitzmann KM (2011) Caregiver awareness of prone play recommendations. Am J Occup Ther 65: 101-105.

18. Koren A, Reece SM, Kahn-D'angelo L, Medeiros D (2010) Parental information behaviors and provider practices related to tummy time and back to sleep. J Pediatr Health Care 24: 222-230.

19. Noritz GH, Murphy NA, Neuromotor Screening Expert Panel (2013) Motor delays: early identification and evaluation. Pediatrics 131: e2016- e2027.

20. Provost B, Lopez B, Heimerl S (2007) A comparison of motor delays in young children: autism spectrum disorder, developmental delay, and developmental concerns. J Autism Dev Disord 37: 321-328.
21. United States Census 2010 (2010) Census data.

22. (2006) Clinical practice guideline: Quick reference guide. Motor disorders assessment and intervention for young children (age 0-3 years). NYS Department of Health Bureau of Early Intervention, Albany, New York.

23. Cohen J, Cohen P, West SG, Aiken LS (2003) Applied multiple regression/ correlation analysis for the behavioral sciences, ( $3^{\text {rd }}$ edn). Lawrence Erlbaum Associates Inc. Publishers, Mahwah, New Jersey.

24. Lobo MA, Galloway JC (2012) Enhanced handling and positioning in early infancy advances development throughout the first year. Child Dev 83: 1290-1302.

25. Carmeli E, Marmur R, Cohen A, Tirosh E (2009) Preferred sleep position and gross motor achievement in early infancy. Eur J Pediatr 168: 711-715.

26. Buonomano DV, Merzenich MM (1998) Cortical plasticity: from synapses to maps. Annu Rev Neurosci 21: 149-186.

27. Nelson CA (1999) Neural plasticity and human development. Curr Dir Psychol Sci 8: 42-45.

28. Lendvai B, Stern EA, Chen B, Svoboda K (2000) Experience- dependent plasticity of dendritic spines in the developing rat barrel cortex in vivo. Nature 404: 876-881.

29. Chechik G, Meilijson I, Ruppin E (1999) Neuronal regulation: a mechanism for synaptic pruning during brain maturation. Neural Comput 11: 2061-2080.

30. Martin JH, Choy M, Pullman S, Meng Z (2004) Corticospinal system development depends on motor experience. J Neurosci 24: 2122- 2132.

31. Bertenthal B, Von Hofsten C (1998) Eye, head and trunk control: the foundation for manual development. Neurosci Biobehav Rev 22: 515- 520.

32. Kleyweg RP, van der Meche FG, Schmitz PI (1991) Interobserver agreement in the assessment of muscle strength and functional abilities in GuillainBarre syndrome. Muscle Nerve 14: 1103-1109.

\section{Acknowledgement}

The authors would like to thank Dr. Elizabeth Poole Di Salvo and Dr. Gail Ross for their thoughtful advice on this article. 